\title{
Transformer Partial Discharge Monitoring Based on Optical Fiber Sensing
}

\author{
Kun WANG, Xinglin TONG*, and Xiaolong ZHU \\ National Engineering Laboratory for Fiber Optic Sensing Technology, Wuhan University of Technology, Wuhan, \\ 430070, China \\ *Corresponding author: Xinglin TONG？ E-mail: 273047432@qq.com
}

\begin{abstract}
The power transformer is the most important equipment of the high voltage power grid, however, some traditional methods of online partial discharge monitoring have some limitations. Based on many advantages of the optical fiber sensing technology, we have done some research on fiber optics Fabry-Perot (FP) sensing which can be useful for the transformer on online partial discharge monitoring. This research aimed at improving the reliability of power system safety monitoring. We have done some work as follows: designing a set for fiber optics FP sensor preparation, according to the fabrication procedure strictly making out the sensors, building a reasonable signal demodulation system for fiber optics FP sensing, doing a preliminary analysis about online partial discharge signal monitoring, including the research on different discharge intensities with the same measuring distance and different measuring distances with the same discharge intensity, and then making a detailed analysis of the experimental results.
\end{abstract}

Keywords: Partial discharge, fiber FP sensor, diaphragm effective reflectivity

Citation: Kun WANG, Xinglin TONG, and Xiaolong ZHU, "Transformer Partial Discharge Monitoring Based on Optical Fiber Sensing," Photonic Sensors, 2014, 4(2): 137-141.

\section{Introduction}

Large amounts of data show that the deterioration of transformer insulation is the most important reason of the fault. At present, the electricity sector adapts the regular maintenance system to prevent and eliminate the breakdown of the transformer. Although the normal operation of the power system has played a certain role, in the maintenance period, interruption of power is needed, the arduous work takes a long time, and the repair process may cause damage to the transformer. These have fully demonstrated the limitations of the off-line maintenance method.

The transformer is the basic equipment which ensures the normal operation of the grid system and reliability of the power supply. Once the faulty happens, it will lead to widespread blackouts that largely influence people's daily life and normal electricity use of productive operation, causing the enormous economic losses. According to a large number of statistics [1-3], the major cause of transformer insulation deterioration is partial discharge. Obviously, the partial discharge comes to be an important sign of deterioration of transformer insulation characteristics. With an online monitoring system of the transformer, we can know the operation status timely, detect the partial discharge, determine a definite location of the insulation defect area, and then repair the transformer promptly. Thus, the research on the partial discharge online monitoring system in this paper is significant and

Received: 10 January 2014 / Revised version: 27 February 2014

(C) The Author(s) 2014. This article is published with open access at Springerlink.com

DOI: $10.1007 / \mathrm{s} 13320-014-0181-4$

Article type: Regular 
valuable.

At present, in order to maintain the excellent insulation characteristics, power transformers are always full of transformer oil inside and equipped with insulation materials such as paperboard. Such design is proved to have the nice mechanical property and electrical strength, but it still has some inherent flaws of inside materials because of the complexity of the manufacturing process. For example, insulation materials have cracks and bubbles, or suspended particle and metal burr in transformer oil, and all such flaws would lead to the partial discharge of the transformer.

Thus, an important symbol of the transformer insulation deterioration is the partial discharge. So, the on-line monitoring of the transformer will be able to reflect the operating conditions of the transformer in real time, detect the partial discharge phenomenon and location insulation defects district, repair the transformer timely and effectively. Studying the online partial discharge monitoring has the important significance and value.

Currently, among the methods of partial discharge online monitoring [4-6], we mainly used piezoelectric and capacitive sensors to detect signals which can be easily interfered by electromagnetic signals. With the advantage of anti-electromagnetic interference, the optical fiber sensing technology can make up for the defects of piezoelectric and capacitive sensors. Meanwhile, in terms of the insulation advantage of the sensing technology, it will become the main transformer safety testing technology.

Nowadays, the fiber optic acoustic emission sensing technology to monitor the partial discharge signal has become a research hot spot [7].

\section{Sensors and sensor systems}

As shown in Fig. 1, in the preparation process, we should firstly bond the quartz diaphragm and quartz sleeve together and then do the same thing to the quartz ferrule and fiber. Secondly, we should place the casing structure and mortise structure in the jib precisely. What is more, by adjusting the jig, we could get the required cavity length and the fringe contrast and then bond the casing structure and mortise structure together. Finally, the production process of the sensor was completed.

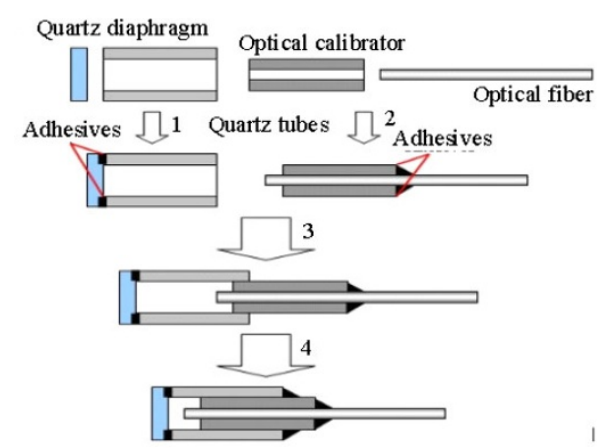

Fig. 1 Flow chart of the preparation technology of the fiber sensor (FP).

In the third process as shown in Fig. 1, we accessed the fiber pigtail into the optical path, connected the broadband source of the super continuum light emission diode (SLED) and spectrometer, and made the instant observation of the interference spectrum. When we got some fixed interference spectrum, we fixed the sensor and got the spectrum which is shown in Fig. 2. According to the analysis of the spectrum in Fig. 2, we could finally get the cavity length and fringe contrast ratio through calculation.

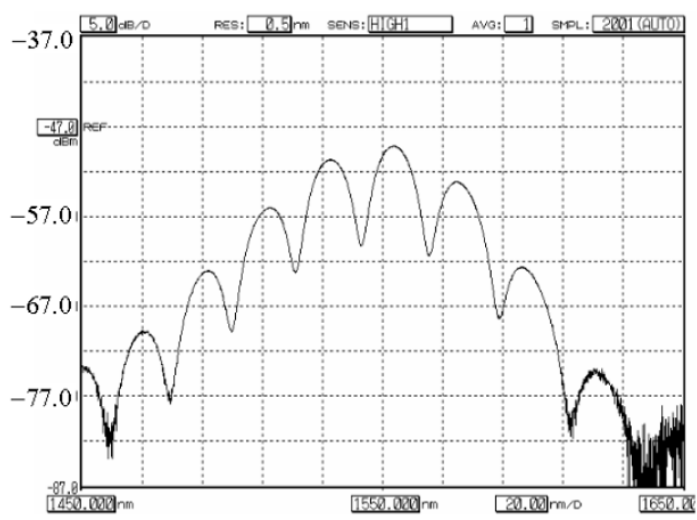

Fig. 2 Interference spectrum of the sensor (the cavity was about $60 \mu \mathrm{m})$.

As shown in Fig. 3, we firstly connected the sensor into one port of the coupler and found the optimal work wavelength through the optical power meter; we changed the wavelength to the best 
working point of the sensor and connected another port of the coupler; then, we connected the demodulator into the last port of the coupler; finally, we connected the digital oscilloscope into the demodulator.

During the experiment, we established the demodulation system which can be seen in Fig. 2 . Instead of measuring the partial discharge signal directly, we used discharge electrode tip discharge signals to generate the partial discharge. Actually, transformer partial discharge signals were transmitted by oil that made the slow signal attenuation. In comparison, the partial generated signal was transmitted by air that made the quick ultrasonic attenuation.

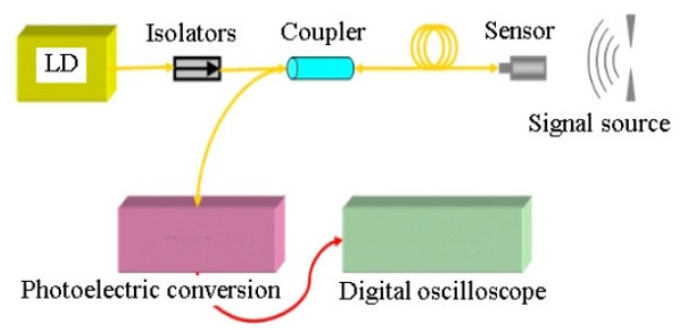

Fig. 3 Pictorial diagram of the sensor demodulation system of the fiber sensor (FP).

\section{Results and discussion}

\subsection{Analysis and experiments of different discharge intensities with the same sensing distance}

In Table 1, the peak voltage can be qualitatively described as the signal intensity of the signal source, indicating that the higher of the peak voltage is, the stronger signal given by the signal source is. The peak frequency can be used to describe the thickness of the quartz diaphragm sensor. The amplitude of the peak frequency can be used to describe the actual detected attenuation time of the sensor. During the attenuation process of the peak voltage from the relatively high position to zero, the higher amplitude means the longer actually detected attenuation time and the slower tendency of attenuation.

According to the data in Table 1: (1) the peak voltage decreases according to the descending order of the discharge signal $(2000 \mathrm{~V}, 420 \mathrm{~V}$, and $400 \mathrm{~V})$, for example, when the peak voltage reaches $2000 \mathrm{~V}$, the discharge signal serves to be most significant, which is consistent with the theoretical analysis; (2) at the vicinity of $350 \mathrm{kHz}$, three groups of peak frequencies tend to be relatively similar, showing that the variable quantity of the cavity length (the phase of $\Delta d)$ is limited to an interval of $(-\pi / 2, \pi / 2)$.

Table 1 Parameters of signal characteristics of the sensor with different discharge intensities.

\begin{tabular}{c|c|c|c}
\hline Discharge signal & $400 \mathrm{~V}$ & $420 \mathrm{~V}$ & $2000 \mathrm{~V}$ \\
\hline Peak voltage (V) & 0.14 & 0.196 & 0.284 \\
\hline Peak frequency (kHz) & 348 & 352 & 348 \\
\hline Amplitude (V) & 77.1 & 40.5 & 81.1 \\
\hline
\end{tabular}

\subsection{Experiment and analysis of the same intensity discharge with different sensing distances}

During this experiment, we used the $2000-\mathrm{V}$ discharge signal as the signal source and the sensor with the cavity length $50 \mu \mathrm{m}$ and contrast ratio $10 \mathrm{~dB}$ to detect the ultrasonic signal excited by signal source. The measured discharge signal waveforms with different sensing distances are shown in Fig. 4.

According to Fig. 5(c), when the sensing distance is $15 \mathrm{~mm}$, after Fourier transformation, the measured data can reach the same peak frequency $(320 \mathrm{kHz})$ as in Figs. 5(a) and 5(b). The reason can be explained that when the sensing distance is above $15 \mathrm{~mm}$, there is the serious attenuation of the discharge signal. As a result, the received signal becomes weak, and the signal distorts because the detected signal is superimposed on the noise, and the errors appear in the Fast Fourier Transform (FFT) analysis of data.

According to the experimental results, the discharge intensity, peak frequency, and amplitude of the measured signal have the negative correlation with sensing distances. The sensing distances and peak voltages, sensing distances, and the amplitudes of response frequencies are separately plotted in Figs. 6(a) and 6(b). 


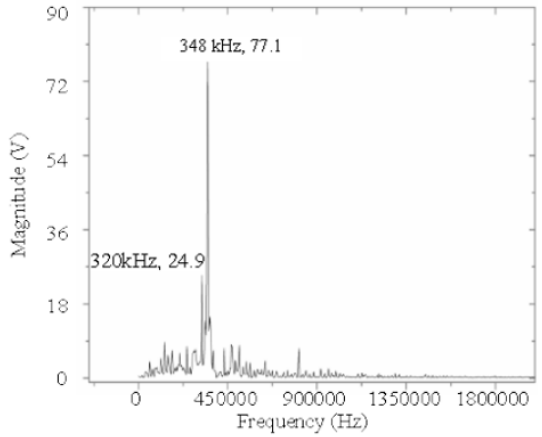

(a) Discharge signal of $400 \mathrm{~V}$

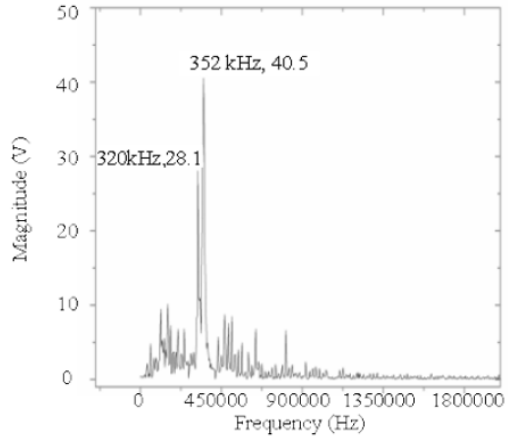

(b) Discharge signal of $420 \mathrm{~V}$

Fig. 4 Spectrogram of the partial discharge signal under three different voltages.

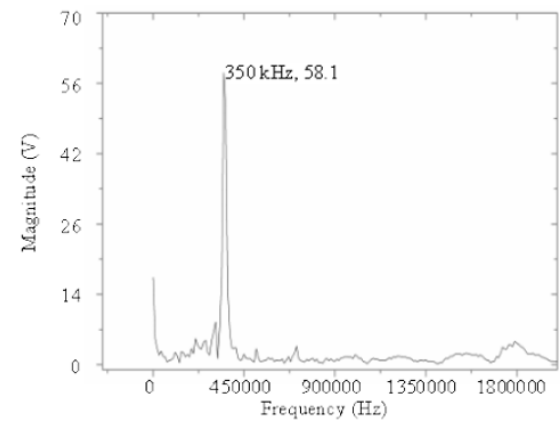

(a) Measured signal at $5 \mathrm{~mm}$

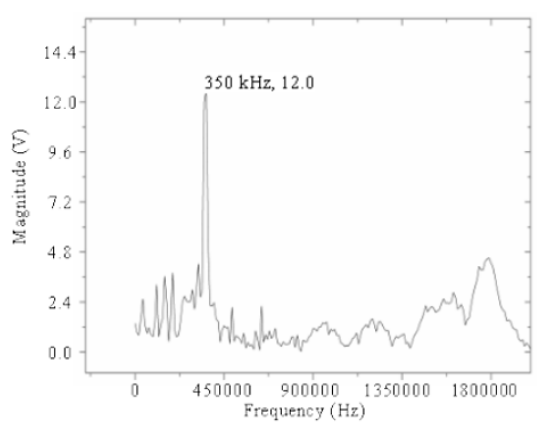

(b) Measured signal at $10 \mathrm{~mm}$

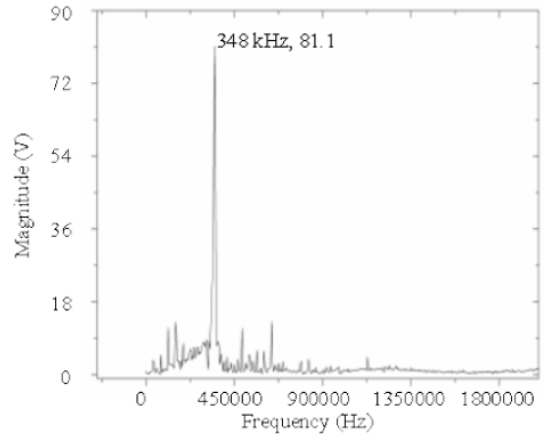

(c) Discharge signal of $2000 \mathrm{~V}$

Fig. 5 Spectrogram of the partial discharge signal with three different sensing distances.

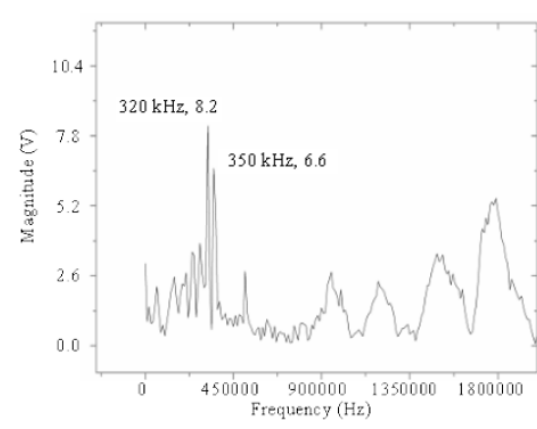

(c) Measured signal at $15 \mathrm{~mm}$

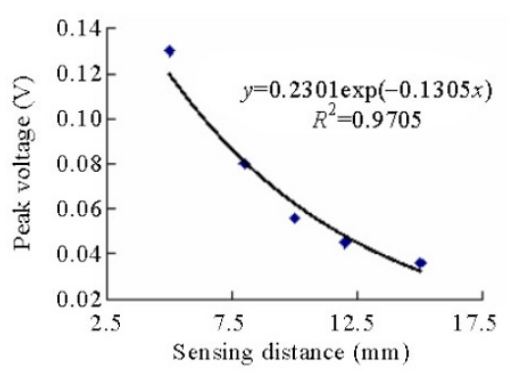

(a)Sensing distance and peak voltage

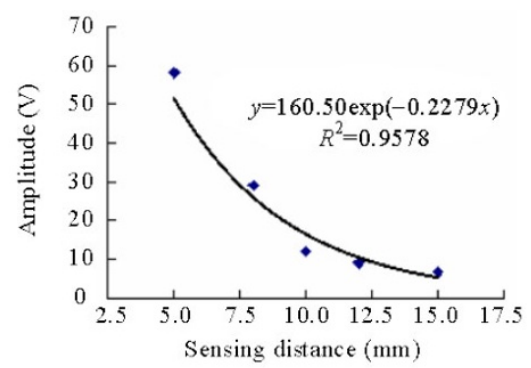

(b)Sensing distance and vibration amplitude

Fig. 6 Relationship between the sensing distance and signal peak voltage.

As shown in Figs. 6(a) and 6(b), with an increase in the sensing distance, the strength of the received signal decays very fast, when the sensing distance surpasses $10 \mathrm{~mm}$, the measured signal becomes not obvious. In order to further improve the sensing distance, we have enhanced the sensitivity of the sensor.

\section{Conclusions}

The power transformer is a very important equipment in the grid operating system, while the existing transformer partial discharge monitoring method has some limitations. Based on many advantages of the optical fiber sensing technology, we did some research on fiber optics FP sensing, which could be used to monitor partial discharge and could do a positive work on improving the reliability of power system safety monitoring.

Due to the limitation of the laboratory, we only made the preliminary research and discussion. There are still many flaws in the production method: stabilization and sensitivity of the sensor, and then the further job includes two parts:

(1) Improve the preparation technology of the sensor: organic glue, which is used in the preparation of the fiber FP sensor in this paper, has the immense influence on the consistency cavity 
length and temperature stability of the sensor. In order to improve the consistency and stability, we can consider using the laser welding technology to make preparation.

(2) Improve the sensitivity of the sensor: the fiber FP sensor in this paper can only detect the signal in a close distance and has a poor sensitivity. In order to improve the sensitivity of the sensor to detect the remote signal without adding its size, we can consider using the thinner-walled tube or diaphragm which is smaller than young modulus materials to make the sensor.

\section{Acknowledgment}

This work was supported by the National Natural Science Foundation of China (Grant No. 51275373) and the Key Project of National Natural Science Foundation of China (Grant No. 50830230).

Open Access This article is distributed under the terms of the Creative Commons Attribution License which permits any use, distribution, and reproduction in any medium, provided the original author(s) and source are credited.

\section{References}

[1] J. Tang, Y. OuYang, M. Fan, X. Zhang, and Y. Liu, "System using fluorescent fiber for partial discharge detection in transformer," High Voltage Engineering, 2011, 37(5): 1129-1135.

[2] J. Tang, J. Zhou, X. Zhang, and F. Liu, "A transformer partial discharge measurement system based on fluorescent fiber," Energies, 2012, 5(5): 1490-1502.

[3] G. C. Stone, "A perspective on online partial discharge monitoring for assessment of the condition of rotating machine stator winding insulation," IEEE of Electrical Insulation Magazine, 2012, 28(5): 8-13.

[4] L. Bao, "Introduction to the on-line transformer PD detection technology," Southern Power System Technology, 2009, 2(6): 81-83.

[5] N. D. Jacob, W. M. Mcdermid, and B. Kordi, "On-line monitoring of partial discharges in a HVDC station environment," IEEE Transactions on Dielectrics and Electrical Insulation, 2012, 19(3): 925-935.

[6] D. Wen, G. Wu, L. Zhou, J. Liu, P. Du, and Y. Liu, "Present status and outlook of PD online monitoring system in power transformer," Electric Age, 2010, 7: 74-77.

[7] D. Xiao, J. Yang, X. Xu, et al., "Fiber optical sensors for partial discharge monitoring in power transformers," Electrical Equipment, 2004(3): 16-18. 